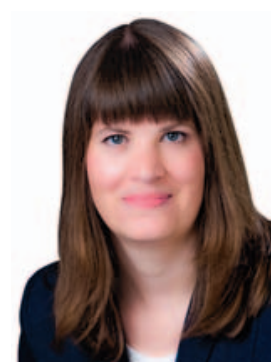

Stephanie Joachim

Koordination «OphthalmoCampus»

\section{Viele Fragen, aber auch viele Antworten: Rechte, Chancen, Möglichkeiten}

Liebe Kolleginnen und Kollegen,

die vierte Ausgabe des «OphthalmoCampus» im Karger Kompass Ophthalmologie beschäftigt in gewohnter Weise mit unterschiedlichsten, sehr interessanten Themen für junge Augenärzte und -ärztinnen.

So haben wir einen Betrag über das Schauspielpatienten-Programm an der Universität Würzburg. Das Programm ermöglicht angehenden Ärztinnen und Ärzten ein hervorragendes und strukturiertes Training von Arzt-Patienten-Gesprächen. Passend dazu gibt Kommunikationstrainerin Dr. Gerlinde Kempendorff-Hoene in einem Interview einen Einblick in ihre Erfahrungen zu Kommunikation im ärztlichen Alltag. Nicht nur die Kommunikation mit Patienten, auch die mit Vorgesetzten oder Kollegen kann den ein oder anderen Fallstrick beinhalten. Ein entsprechendes Kommunikationstraining kann hier weiterhelfen. Ein weiterer Beitrag, der für Augenärztinnen von großem Interesse sein wird, beschäftigt sich dem Arbeiten während der Schwangerschaft. Ein Thema, das nicht nur für operativ tätige Augenärztinnen relevant ist.

Als Abschluss stellt uns Frau Vollmer das Buch «Time and Life Management for Medical Students and Residents» von Michael Sabel vor. Bei den zahlreichen Aufgaben, mit denen wir während der Weiterbildung beschäftig sind, ist eine gute Organisation des Berufsalltags und ein entsprechendes Zeitmanagement eminent wichtig.

Bei der Lektüre des aktuellen «OphthalmoCampus» wünsche ich viel Vergnügen!<smiles>CCCCC(C)OCC</smiles>

\section{KARGER}

Fax +497614520714

information@karger.com www.karger.com (c) 2017 S. Karger GmbH, Freiburg
PD Dr. Stephanie Joachim

Head of Experimental Eye Research

In der Schornau 23-25, 44892 Bochum, Deutschland

stephanie.joachim@rub.de 


\section{Schauspielpatienten-Programm an der Medizinischen Fakultät der Universität Würzburg}

Krankheitsverlauf, Adhärenz, Behandlungserfolg und nicht zuletzt eine gelungene Kommunikation [1] sind Aspekte, die eine gute Arzt-PatientenBeziehung positiv beeinflussen. Um den Grundstein für diese essenziellen kommunikativen Fähigkeiten möglichst bereits im Medizinstudium zu legen, werden weltweit an zahlreichen Universitäten Schauspielpatienten eingesetzt, d.h. Laienschauspieler, die für bestimmte Patientenrollen trainiert werden: Dies umfasst nicht nur eine medizinische Vorgeschichte mit Symptomen, Voruntersuchungen usw., sondern auch nicht-medizinische Informationen wie den Berufoder diefamiliäre Situation des erdachten Patienten. Ziel ist es, einen möglichst authentischen Patienten entstehen zu lassen. Seit 2007 gibt es das Schauspielpatienten-Programm an der Universität Würzburg. Wir hatten die Möglichkeit, mit Dr. Matthias Lukasczik und Dipl.-Psych. Hans-Dieter Wolf, den beiden Koordinatoren des Programms, zu sprechen.

\section{Wie läuft ein Schauspielpatientengespräch ab?}

Die Gespräche finden in Kleingruppen von maximal 12 Personen statt und dauern jeweils ca. 10 min. Sie werden in den Lehrveranstaltungen vorbereitet, d.h. die Dozenten erarbeiten mit den Studenten allgemeine Informationen und geben Input zur Situation und zu den Fertigkeiten, die für ein gutes Gespräch wichtig sind. Außerdem erhalten die Studenten eine schriftliche Kurzinformation mit Angaben zum Patienten, zu medizinischen Fakten und zu ihrer Aufgabe als Arzt im Gespräch.

Im Gespräch selbst sind jeweils ein Dozent bzw. Tutor sowie die anderen Mitglieder der Kleingruppe anwesend. Nach dem Gespräch folgt ein strukturiertes Feedback: Nachdem der Student, der die Arztrolle übernommen hat, seine Einschätzung des Gesprächs

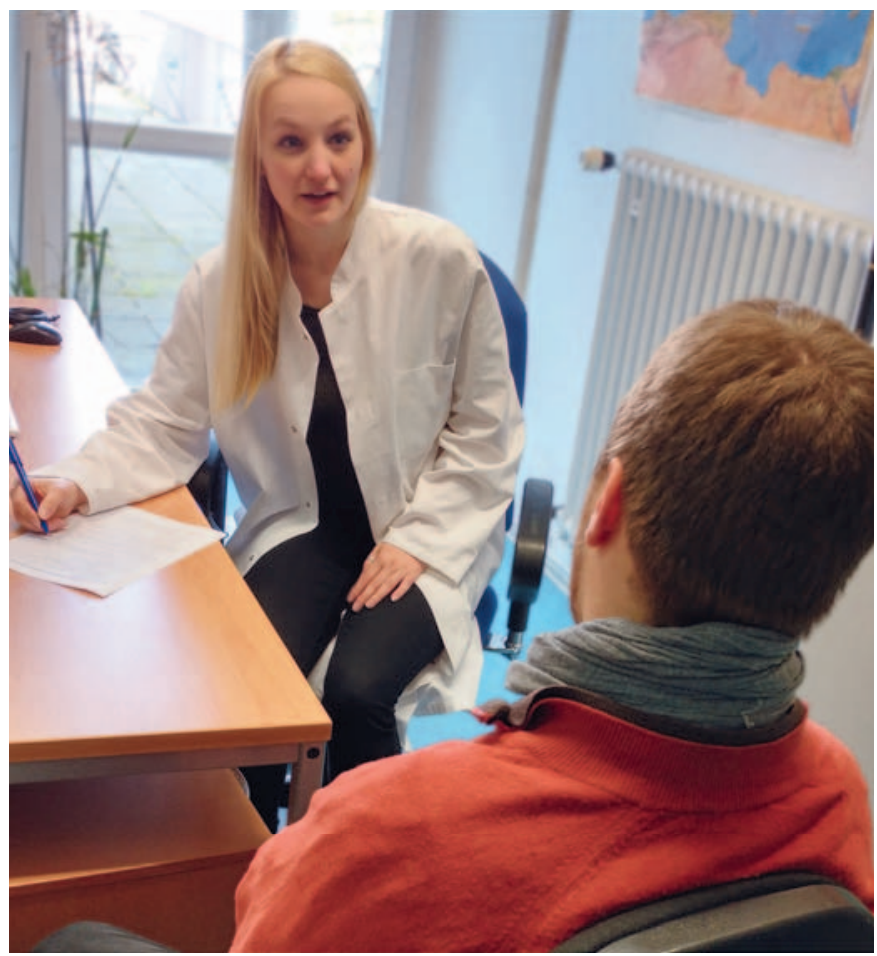

${ }^{\circledR}$ Matthias Lukasczik, Schauspielpatienten-Programm Würzburg. abgegeben hat, äußert sich der Schauspielpatient (SP) dazu, was im Gespräch gut gelaufen ist und an welchen Stellen es Verbesserungsbedarf gibt. Danach geben der Dozent und die anderen Kursteilnehmer Rückmeldung.

\section{Wie wird eine Patientenrolle kreiert?}

Zunächst überlegen wir uns, wo Bedarf für eine neue Rolle besteht. Hieraus leiten sich in der Regel schon einige Vorgaben ab (Erkrankung, Lebensstil des Patienten, vermutliche Gefühlslage usw.).

Mithilfe dieses Grundgerüstes folgt in der Regel zuerst eine Art Brainstorming mit den für das jeweilige Fach verantwortlichen Dozenten. An der detaillierten Formulierung der Rolle sind immer mehrere Personen aus verschiedenen Berufsgruppen (Psychologen, Ärzte) beteiligt.

Die Rolle umfasst im Ergebnis dann mindestens die folgenden Informationen:

- Angaben zur Person (Name, Alter inkl. Geburtsdatum, Familie)

- Angaben zur beruflichen Situation und Laufbahn

- Angaben zur aktuellen medizinischen Situation

- Angaben zur medizinischen Vorgeschichte

- Angaben zu Persönlichkeit und sozialer Situation

Die so entstandene Rolle wird dann mit einem oder mehreren SP durchgearbeitet und im Rollentraining praktisch getestet. Input von Schauspielerseite wird aufgegriffen, mögliche Unklarheiten oder «Stolpersteine» werden berücksichtigt und die Rolle entsprechend angepasst. Erst dann kann sie in der Lehrroutine eingesetzt werden.

Gibt es «schwierige» Rollen, die beispielsweise den kulturellen Hintergrund und die damit verbundenen Herausforderungen berücksichtigen?

Es gibt Rollen, die aufgrund ihrer medizinischen Situation herausfordernd sind - dazu zählt z.B. die Rolle einer jungen Mutter mit fortgeschrittener Krebserkrankung, die aller Wahrscheinlichkeit nach ihr Kind nicht aufwachsen sehen wird. Andere sicher sinnvolle und spannende Konstellationen, z.B. Angehörigengespräche oder Rollen aus anderen kulturellen Hintergründen, sind Teil unserer langfristigen Planungen, bislang aber noch nicht umgesetzt.

Welche Problemfelder/Herausforderungen haben sich im Laufe der Jahre herauskristallisiert und was kann man daraus für die Praxis lernen?

Eine der größten Schwierigkeiten, mit der gerade junge Mediziner zurechtkommen müssen, ist der immer größer werdende Zeitdruck im ärztlichen Berufsalltag. Eine der großen Herausforderungen ist es daher, zu zeigen, dass man auch in zehn Minuten eine gute Beziehung zu einem Patienten aufbauen und auf seine Bedürfnisse eingehen kann.

Aufgrund der demographischen Entwicklung nimmt auch die Arbeit mit älteren Menschen einen immer größeren Raum in der ärztlichen Arbeit ein. Deshalb sind mehrere Rollen im Würzburger SP- 
Programm bereits auf ältere Patienten zugeschnitten, um den Studierenden das Üben alltagsrelevanter Gesprächssituationen zu ermöglichen.

Welche Entwicklungen gab es in den nunmehr 10 Jahren, in denen das Programm schon läuft und welche Pläne gibt es für die Weiterentwicklung des Programms?

In erster Linie ist das Programm ausgebaut und erweitert worden - sowohl was den Pool an Rollen als auch was die Zahl der Lehrveranstaltungen mit SP-Einsätzen betrifft. Mittlerweile ist die Arbeit mit SP ein fester Bestandteil der medizinischen Lehre in Würzburg und wird von den Studenten sehr gut bewertet. Der hohe Zuspruch zeigt sich auch an den vielen Interessierten, die sich als Tutoren bzw. als SP bewerben. Die gute Entwicklung des Programms findet fächerübergreifend Anerkennung und wurde 2011 mit dem AlbertKölliker-Lehrpreis der Medizinischen Fakultät ausgezeichnet. Ein Faktor, der für die medizinische Ausbildung sicher relevant ist, ist der Nationale Kompetenzbasierte Lernzielkatalog Medizin (NKLM). Dort wird Kommunikationsfertigkeiten ein großer Stellenwert beigemessen. Daher wird sicher die Frage nach der Prüfbarkeit der erlernten Kommunikationskompetenzen, z.B. im Rahmen von OSCE-Prüfungen, noch mehr in den Vordergrund rücken. Hier steht das SP-Programm in Würzburg noch in der Planungsphase.

Wir danken Ihnen herzlich für das Interivew!
Angaben zum Schauspielpatienten-Programm in Würzburg Das Programm wurde 2007 von Ärzten und Psychologen des Uniklinikums (Palliativmedizin) und der Universität (Medizinische Psychologie) initiiert, damals noch beschränkt auf den Bereich Onkologie. Aktuell werden Schauspielpatienten in vier Lehrveranstaltungen eingesetzt: «Medizinische Psychologie» (Anamnese, Motivierung zur Lebensstiländerung, Aufklärung/ Diagnosemitteilung), «Praktische Klinische Untersuchungsmethoden»(Anamnese), «Kommunikationstraining Interdisziplinäre Onkologie» (Diagnosemitteilung) und «OP-Aufklärung». Insgesamt kommen derzeit ca. 30 Schauspielerinnen und Schauspieler in 20 verschiedenen Rollen zum Einsatz.

Nähere Informationen finden Sie unter www.psychotherapie.uni-wuerzburg.de/schauspielpatienten

\section{Literatur}

1 Interview mit Samy Molcho: Von Mensch zu Mensch statt von Arzt zu Patient. Karger Kompass Dermatol 2017:5;98.

Kontaktadresse: Dr. Matthias Lukasczik / Dipl.-Psych. Hans-Dieter Wolf, JuliusMaximilians-Universität Würzburg, Abteilung für Medizinische Psychologie, Klinikstraße 3, 97070 Würzburg, Deutschland, matthias.lukasczik @ uniwuerzburg.de; hans.wolf@uni-wuerzburg.de.

\section{Arzt-Arzt-Kommunikation}

«Frauen reden anders, Männer auch. Gut zu wissen.»

Dr. Gerlinde Kempendorff-Hoene liegt das Thema Kommunikation sehr am Herzen. Aus diesem Engagement heraus gründete sie 2012 das «Kempendorff Privatinstitut für Kommunikation und Kultur» (KIKK). Als Coach für Sprechkultur und Auftrittskompetenz ist sie seit 2012 unterwegs und das gibt ihr regelmäßig die Möglichkeit, in verschiedene Fachgebiete hineinzuschnuppern - unter anderem auch in die Medizin.

Sie empfehlen für den zwischenärztlichen Kontakt das 5-SäulenSystem der Rhetorik. Was ist das Besondere diesem System?

Das 5-Säulen-System der Rhetorik von Christian Rangenau ordnet einfach mal alles, was - übrigens fachübergreifend - zur gelingenden Kommunikation gehört, in ein übersichtliches System. Daraus ergibt sich für den Laien erst einmal das Verständnis dafür, worauf geachtet werden muss und was gearbeitet, geübt und damit auch geändert werden kann. Vielen Menschen ist nicht klar, dass die Rhetorik ein eigenes Fachgebiet ist. Damit lässt sich ins Bewusstsein rücken, dass man durchaus eine «berufliche» und eine «private» Sprache pflegen kann, wobei in der ersteren gegenseitiger Respekt, Wertschätzung und Sachlichkeit immer im Vordergrund stehen müssen.

Auf welche geschlechtsspezifischen Unterschiede in der Kommunikation sollte man gerade im medizinischen Arbeitsalltag achten?

Die Sprache ist immer im Wandel, und mit der Gleichstellung von Mann und Frau in der Sprache geschieht gerade eine größere Veränderung, als mancher vertragen kann. Dazu kommen auch
Übertreibungen, die Sachverhalte verwässern. Ich finde es angenehm, wenn man z.B. in Vorträgen oder Veröffentlichungen Gattungs- und Genusbegriff klar trennt und das im Vorfeld deutlich macht. Der Gattungsbegriff ist «Mensch», der Genusbegriff weiblich, männlich oder sächlich, d.h. wenn ich sage: «Der Mensch hat unterschiedliche Arten zu kommunizieren», muss ich nicht hinzufügen «die Menschin», denn der Begriff Mensch beinhaltet alle Geschlechter. Wenn ich aber sage: «Chefarzt Bettina Müller», sollte sich jeder daran gewöhnen, den weiblichen Begriff «Chefärztin» zu benutzen. Auch die - leider immer noch gängige - Anrede von PatientInnen mit «Was haben wir denn?» sollte langsam mal unterlassen werden. Ich antworte dann immer: «Was Sie haben, weiß ich nicht, aber ich habe ...».

Welche Kommunikationstipps geben Sie Ärztinnen, die am Beginn ihrer Karriere stehen, mit auf den Weg?

Kleine Geschichten aus dem Arbeitsalltag machen das sehr deutlich.

- Eine junge Assistenzärztin war bei mir im Training, weil sie sich über ihren Chef geärgert hatte oder viel mehr über sich und ihre Hilflosigkeit. Während der Visite begegnete der Ärzteschar auf dem Gang eine ältere Patientin, die offensichtlich Hilfe benötigte, und der Chefarzt sagte zu der Patientin, mit einem Kopfnicken zur Assistenzärztin deutend: «Die Anne bringt sie mal auf das Zimmer!» Damit war den anderen Kolleglnnen klar, dass er sie nicht als Kollegin wahrnimmt und der Patientin wurde vermit- 
telt, dass sie eine Schwester sein muss. Und: Sie hat sich nicht getraut, dem Cheffreundlich aber bestimmt diesen Fauxpas aufzuzeigen, den er wahrscheinlich gar nicht gemerkt hatte. Viele, gerade junge MedizinerInnen haben im immer noch sehr hierarchischen Denken (auch von PatientInnenseite) Akzeptanzschwierigkeiten. Das muss sich ändern. Dazu brauchen sie rhetorisches Geschick, das man erlernen kann.

- Eine junge Augenchirurgin erzählte mir, dass oft PatientInnen zu ihr sagen: «Wann kommt denn nun der Doktor?!». Sie war dann immer ärgerlich und wir haben erarbeitet, dass sie von nun an lächelnd sagt: «Der bin ich, und was Besseres kann ihnen nicht passieren.»

Gerade Frauen haben oft das Problem, sich emotional angegriffen zu fühlen, wo ihnen eigentlich nur nachlässiges und/oder altmodisches Denken entgegentritt. Das kann man sich abtrainieren, damit die eigene Zufriedenheit wächst und Job und Karriere gedeihen können.

Frau Dr. Kempendorff-Hoene, vielen Dank für dieses Gespräch!

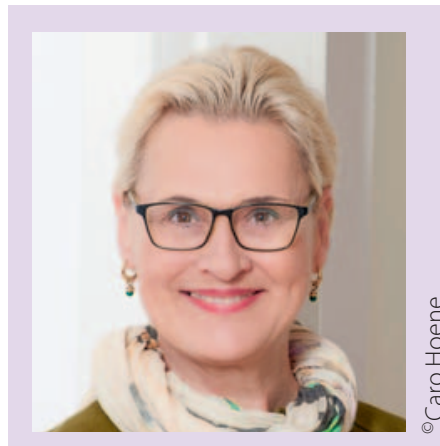

Dr. Gerlinde Kempendorff-Hoene
ZurPerson

Nach ihrer freiberuflichen Tätigkeit als Kabarettistin, Sängerin, Moderatorin und Dozentin arbeitete Frau Dr. Gerlinde Kempendorff-Hoene seit 1991 als Dozentin an der Universität der Künste Berlin. 2012 machte sie sich mit der Gründung des Kempendorff Privatinstituts für Kommunikation und Kultur (KIKK) selbstständig. Seit über 30 Jahren in der Erwachsenenqualifizierung tätig, konzentriert sie sich seit 5 Jahren auf die Arbeit als Coach für Auftrittskompetenz und Sprechkultur.

\section{Weiterführende Literatur}

Kempendorff-Hoene G: Lehrer und Kabarettisten, ed 1. Berlin, Lehmanns Media, 2010

Kontaktadresse: Dr. phil. Gerlinde Kempendorff-Hoene, Büro Kempendorff, Leibnizstraße 35, 10625 Berlin, Deutschland, www.sprechkultur-kikk.de.

\section{Arbeitsrecht}

\section{Schwangerschaft in der Weiterbildung}

Viele junge Ärztinnen machen ihre Facharztweiterbildung im Alter zwischen 25 und 35 Jahren, also genau dann, wenn oftmals die Familienplanung konkret wird. Was ist beieiner Schwangerschaft und der Elternzeit zu beachten?

Kliniken geraten immer mehr unter Druck, familienfreundliche Lösungen wie Teilzeit, Gleitzeit oder Kinderbetreuung anzubieten, weil derzeit 60\% der Medizinstudierenden Frauen sind und es folglich immer mehr Ärztinnen geben wird. Bei vielen Kliniken lassen familienfreundliche Maßnahmen aber noch zu wünschen übrig Deshalb sollte man bei der Bewerbung berücksichtigen, inwiefern der potenzielle Arbeitgeber mit einer Halbtagstätigkeit, mit einem Betriebskindergarten etc. entgegenkommt.

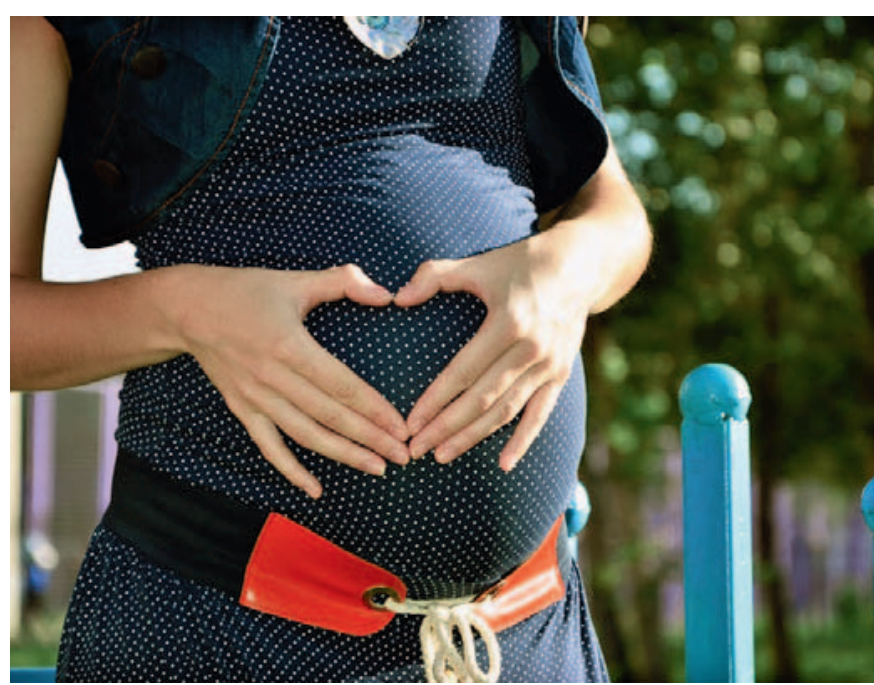

\section{No-Gos in der Schwangerschaft}

Es gibt verschiedene gesetzliche Regelungen, die schwangere Ärztinnen und ihr ungeborenes Kind vor gesundheitlichen Schäden und Überforderung am Arbeitsplatz schützen sollen. Insbesondere im Mutterschutzgesetz und in der Mutterschutzrichtlinienverordnung ist festgelegt, was man als werdende oder stillende Mutter nicht mehr machen darf. Dazu gehört u.a.:

- Nachtarbeit zwischen 20:00 und 6:00 Uhr

- Bereitschaftsdienste

- Notdienste

- Mehrarbeit

- regelmäßiges Heben von mehr als $5 \mathrm{~kg}$

- Kontakt zu giftigen und gesundheitsschädlichen Gefahrenstoffen

- Kontakt zu potenziell infektiösem Material

- Injektionen, Punktionen, Blutentnahmen, Operationen.

Es empfiehlt sich, die Schutzverordnungen genau durchzulesen, damit man weiß, auf welche Rechte man sich berufen kann. So ist der Arbeitgeber z.B. auch verpflichtet, einer schwangeren Ärztin einen Ruheraum zur Verfügung zu stellen und die Schwangerschaft beim zuständigen Landesamt für Arbeitsschutz und Gesundheitsschutz zu melden. Kann der Arbeitgeber nicht alle Maßnahmen umsetzen, die einer schwangeren Ärztin zustehen, muss der Betriebsarzt ein Beschäftigungsverbot bescheinigen. Man bleibt dann zu Hause, bezieht das Gehalt aber weiter.

Die Richtlinien zum Schutze von Mutter und Kind werden aber auch kritisiert, zum Beispiel vom Deutschen Ärztinnenbund (DÄB), weil der schwangeren Ärztin der Patientenkontakt aufgrund der Bestimmungen mehr oder weniger verboten wird. Die Bestimmungen sind z.T. schon 20 Jahre alt, die OP-Technik hat sich 
in dieser Zeit aber stark weiterentwickelt. Das in der Richtlinie festgelegte generelle OP-Verbot erscheint vielen als zu hart. Derzeit haben schwangere Ärztinnen nur zwei Möglichkeiten: Entweder sie teilen ihre Schwangerschaft frühzeitig mit. Dann müssen sie damit rechnen vom Dienst freigestellt zu werden und können keine weiteren Leistungen für die Weiterbildung sammeln. Oder: Sie machen die Schwangerschaft erst sehr spät öffentlich. Dann genießen sie keinen gesonderten Schutz und werden von den unwissenden Kollegen womöglich wegen häufiger Ruhezeiten kritisiert.

\section{Mutterschutzbestimmungen}

Bis es zu einer überarbeitenden Mutterschutz-Richtlinie für Ärztinnen kommt, muss man als schwangere Ärztin also selbst überlegen, wie man mit der eigenen Schwangerschaft umgeht. Am besten bespricht man sich gründlich mit dem Partner und der Frauenärztin (auch sie darf ein Berufsverbot aussprechen). Oft sagt einem auch der eigene Körper, was er braucht.
Wenn man in der Schwangerschaft arbeitet, besteht die Möglichkeit, sechs Wochen vor dem errechneten Geburtstermin in den «Mutterschutz» zu gehen, also nicht mehr zu arbeiten. Nach der Geburt ist man 8 Wochen verpflichtend im Mutterschutz. Während dieser Zeit bekommt man ein Mutterschaftstagegeld von der Krankenkasse, zusätzlich überweist einem der Arbeitgeber die Differenz zwischen Mutterschaftsgeld und dem vorherigen Nettogehalt. Während des Mutterschutzes bekommt man also das gleiche Geld überwiesen wie in der Zeit davor und muss keine finanziellen Einbußen hinnehmen.

\section{Originalquelle}

Lukas Hoffmann für Operation Karriere - Das Karriereportal und der Kongress für angehende und junge Ärzte, www.operation-karriere.de $\rightarrow$ Karriereweg $\rightarrow$ Assistenzarzt (Direktlink für Onlineversion: www.operation-karriere.de/karriereweg/assistenzarzt/ schwangerschaft-in-der-weiterbildung.html).

opperaron

\section{Buchrezension}

\section{Michael Sabel: Time and Life Management for Medical Students and Residents}

Stuttgart, Thieme, 2017, 76 Seiten, 24,99 EUR

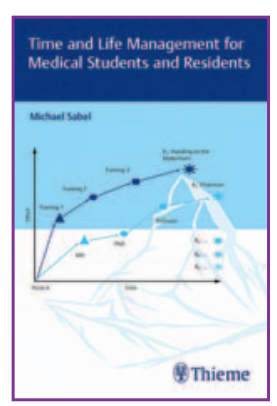

Das Buch von Herrn Sabel beschäftigt sich mit einem Thema, das im Medizinstudium leider keinen Platz gefunden hat und doch sehr wichtig ist. Die zentrale Frage lautet: Wie lerne ich mich selbst zu organisieren und meine Ziele zu erreichen?

Das Buch beginnt mit der Aufforderung, sich erst einmal über seine Ziele und Ambitionen klar zu werden. Hierzu werden verschiedene kleine Übungen und Denkexperimente vorgeschlagen. Das Buch wird erst dann zu einer wertvollen Hilfe, wenn diese Übungen auch tatsächlich durchgeführt werden, denn alle folgenden Kapitel setzen klar gesteckte Ziele voraus. Ist man sich über seine Ziele bewusst geworden, so geht es im zweiten Abschnitt darum, wie man diese erreichen kann. Wie kann ich mich motivieren meine Ziele zu verfolgen und nicht aufzugeben? Wie entscheide ich, welche Aufgaben wirklich wichtig sind? Es geht in diesem Buch um das, was wohl die meisten Medizinstudenten nach dem Studium qualvoll lernen müssen: den Alltag auf Station effektiv zu gestalten, sich auch einmal abzugrenzen und die nötige Balance zwischen Arbeits- und Privatleben zu finden. Konkrete Vorschläge, wie man seine Effektivität dann tatsäch- lich erhöhen kann, liefert das Buch meiner Meinung nach leider nur eingeschränkt, bleibt es doch häufig bei allgemeinen Grundsätzen. Die vielen Graphiken verdeutlichen die im Text beschriebenen Theorien sehr plastisch, liefern aber keine neue Aussage und waren mir zu trivial. Für Menschen, die sich bereits mit dem Thema «Selbstmanagement» beschäftigt haben, mag das Buch augenscheinlich keine neuen Erkenntnisse bieten. Außerdem fehlte mir ein stringenter Aufbau. Alle Kapitel waren zwar in sich schlüssig aufgebaut, jedoch fehlte der übergeordnete Zusammenhang zwischen den Kapiteln.

Trotz allem hat das Buch seine Daseinsberechtigung, denn eines tut es sicher: Es regt zum Denken an. Was für ein Arzt möchte ich sein? Was für ein Mensch möchte ich sein? Wo liegen meine Prioritäten? Sind meine Ziele realistisch und nutze ich meine Zeit sinnvoll? Sich bereits vor Arbeitsbeginn einmal mit diesen Fragen auseinanderzusetzen spart in der ohnehin stressigen Anfangszeit sicherlich viel Zeit und Kraft.

Clara Vollmer, Kiel

Kontaktadresse: Clara Vollmer, Klinik für Innere Medizin IV, Nieren- und Hochdruckkrankheiten, Universitätsklinikum Schleswig-Holstein, Schittenhelmstraße 12, 24105 Kiel, Deutschland, claracvollmer@googlemail.com. 